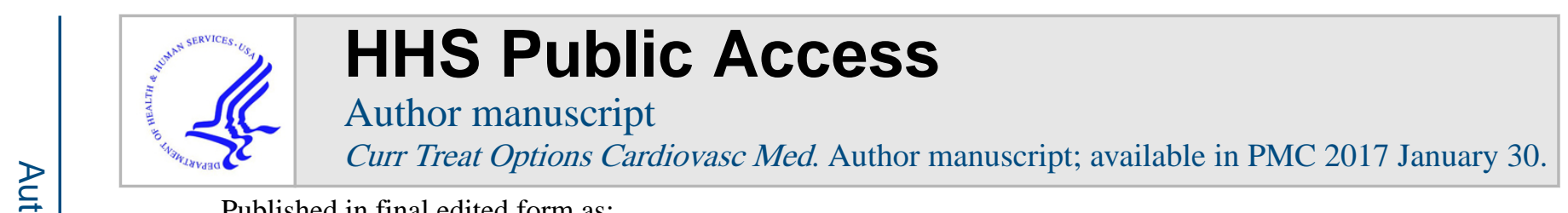

Published in final edited form as:

Curr Treat Options Cardiovasc Med. 2016 September ; 18(9): 58. doi:10.1007/s11936-016-0480-8.

\title{
Direct Cardiac Cellular Reprogramming for Cardiac Regeneration
}

\author{
Vivekkumar Patel, MD, Megumi Mathison, MD, PhD, Vivek P. Singh, PhD, Jianchang Yang, \\ MD, PhD, and Todd K. Rosengart, MD
}

\author{
Keywords \\ Gene therapy; Heart failure; Cardiomyocyte; Reprogramming; Transdifferentiation; GMT
}

\section{Introduction}

Despite significant advances in the treatment of individuals with advanced heart failure, including increasing survival rates associated with heart transplant and mechanical circulatory support, a great unmet need exists to improve the quality and duration of the lives of patients with this condition affecting over one million Americans [1,2]. The implantation of exogenous stem cell has for over a decade been the focus of efforts to "biologically" enhance heart function in patients with congestive failure, but ineffectual clinical applications of such strategies has fueled disenchantment with this cell delivery approach [3•]. The recently discovered capability to utilize various combinations of cellular reprograming transcription factors and other agents to directly transdifferentiate fibroblasts into (induced) cardiomyocytes (iCMs) offers the intriguing potential to convert abundant numbers of scar fibroblasts found in myocardial infarction zones into iCMs as a means of improving post-infarct ventricular function $[4,5]$. Such a strategy would allow in situ cardiac cellular reprogramming without the need for exogenous cell implantation.

\section{Options for myocardial regeneration}

The concept of cellular reprogramming emerged from Dr. Yamanaka's innovative discovery that terminally differentiated cells may be reprogrammed into induced pluripotent stem (iPS) cells following overexpression of key transcription factors Oct4, Sox2, Klf4, and c-myc [6••]. Subsequently, Srivastava et al. discovered that three key factors, Gata4, Mef2c, and Tbx5 (GMT), were capable of directly reprogramming cardiac fibroblasts into contractileinduced cardiomyocytes (iCMs), bypassing the intermediate pluripotent state in a process called "transdifferentiation" [7••]. Encouragingly, intramyocardial injection of these

\footnotetext{
*Michael E. DeBakey Department of Surgery, Baylor College of Medicine, 6501 Fannin Street, Suite NC114, Houston, TX, 77030, USA, Todd.Rosengart@bcm.edu.

Compliance with Ethical Standards

Human and Animal Rights and Informed Consent

This article does not contain any studies with human or animal subjects performed by any of the authors.

Conflict of Interest

The authors declare that they have no conflicts of interest.
} 
reprogramming factors has led to a significant improvement in post-infarct ventricular function in rodent models [8•, 9-15].

Direct cardiac cellular reprogramming may present significant advantages over other cardiac regenerative techniques, such as the implantation of stem cells (bone marrow derived or induced pluripotent), induction of existing cardiomyocyte proliferation, or engraftment of engineered tissue embedded with iPS cells, none of which have yet produced compelling outcomes in clinical trials. More specifically, the implantation of stem cells derived from bone marrow or other sources has failed to achieve clinically significant improvements in cardiac function in a variety of clinical trials $[3 \bullet, 16]$. These discouraging outcomes are thought to be related to inefficient cell delivery, low cell survival in an ischemic milieu, poor engraftment into native myocardium, and incomplete development by stem cells of cardiomyocyte structures [17•].

If exogenous cell implants are derived from an allogeneic source, there is an additional concern of immunorejection, which may necessitate immunosuppressive medication. The risk of arrhythmia and the potential for teratoma formation, particularly for iPS cells, are other liabilities associated with exogenous cell delivery [17•, 18]. Finally, while the implantation of artificial tissue grafts embedded with stem cells or even engineered cardiomyocytes derived from iPS cells have been tested as a means of enhancing exogenous cell engraftment, many of the same barriers exist to the success of this strategy as are found in non-embedded cell implantation strategies, including poor electrical integration of graft cells into the host myocardium [19].

In the context of the normally exceedingly infrequent incidence of adult cardiomyocyte replication $(<1 \%)$, the possibility of inducing resident cardiomyocyte proliferation through modulation of cell cycle reentry represents an alternative strategy to biologically enhance post-infarct myocardial function [20,21]. While in vitro and small animal testing of this strategy are encouraging, clinical trials to induce cardiomyocyte replication have not yet begun and may potentially be hindered by the risk of promiscuous or excessive cell replication resulting in tumorigenesis [21].

\section{Direct cardiac cellular reprogramming}

Initial studies of direct cellular reprogramming reported by Srivastava as well as others noted reprogramming efficiencies only in the range of approximately $7 \%$ of treated fibroblast target cells, as assessed by the expression by these cells of cardiomyocyte-specific markers such as cardiac Troponin T (cTnT) [7••, 22]. An even smaller proportion $(<1 \%)$ of cells treated with reprogramming factors were found to be contractile, although this number could for example be enhanced by co-culturing with embryonic cardiomyocytes, presumably on the basis of exposure to an extracellular milieu more conducive to cardiodifferentiation [23].

Following on these initial reports, a growing number of subsequent studies attempted to increase the efficiency and quality of reprogramming through the addition of enhancing cardio-differentiating factors, or downregulation of genes that regulate the native fibroblast 
signature, with varying degrees of effectiveness (Table 1). These efforts were largely based upon the addition of various potential cardiac-differentiating factors that were derived from analyses of relevant drivers of embryologic cardiac differentiation.

Song et al. for example reported that addition of Hand2 to GMT enhanced the efficiency of reprogramming by threefold, as evidenced by the percentage of $\mathrm{cTnT}^{+}$cells identified by flow cytometry (i.e., $\sim 25 \%$ of cells were cTnT $^{+}$after treatment with GMT + Hand 2 vs $\sim 10 \%$ after GMT treatment) [10]. Similarly, Addis et al. reported that addition of Nkx2.5 and Hand2 to GMT was nearly 50-fold more efficient than GMT alone, based on calcium activity [28]. Others confirmed that Myocardin not only enhanced reprogramming but could also replace other reprogramming factors, thereby reducing the total number of factors needed to induce reprogramming [29-31]. Taken together, the addition of select cardiodifferentiating factors to GMT was found to enhance the generation of a cardiomyocyte phenotype from cardiomyocytes, as evidenced by improved reprogramming efficiency and the functional characteristics of iCMs.

Optimizing the "dosage" of GMT or other reprogramming factors has also been pursued as a means of enhancing reprogramming efficiency. For example, as compared to the use of "singlet" vectors encoding individual transgenes, we and others have demonstrated that use of a "triplet" vector encoding all three GMT reprogramming factors resulted in greater reprogramming efficiency (presumably because target cells needed to be infected with only one rather than three vectors), more mature development of sarcomeric structures (e.g., as demonstrated by a-actinin immunofluorescence), and nearly a threefold greater increase in post-infarct ventricular function $[14,15]$.

Two other studies further confirmed that differential overexpression of Mef2c in relation to Gata4 and Tbx5 results in more rapid and increased formation of contractile iCMs [24, 26]. Specifically, Wang et al. demonstrated that stoichiometric rearrangement of a GMT triplet retroviral vector to MGT increased Mef2c expression and was associated with a 10-fold increase in the number of contractile iCMs [24].

Other studies suggest that suppression of fibroblast gene expression may be as important as optimization of cardio-differentiating factors in enhancing iCM reprogramming efficiency. Muroaka et al. elegantly demonstrated a sixfold enhancement of reprogramming following microRNA 133 (miR-133)-mediated downregulation of Snai1, a master regulator of epithelial-to-mesenchymal transition and promoter of profibroblast gene expression [38]. Downregulation of the fibroblast signature by targeting transforming growth factor- $\beta$ (TGF $\beta$ ) or Rho-associated kinase pathways has similarly been shown to enhance the efficiency and quality of cardiac reprogramming [32, 33].

While most of the aforementioned approaches have used integrative retroviral or lentiviral vectors to deliver reprogramming genes to target cells, several other investigators have employed transient expression of miRNA or small molecules to, respectively, induce transdifferentiation via non-integrative genetic strategies and non-genetic strategies altogether. Jayawardena et al. for example demonstrated that transient transfection of miR-1, miR-133, miR-208, and miR-499 can reprogram fibroblasts into iCMs albeit at a low 
efficiency [12]. We likewise recently demonstrated that miR-590 could enhance GMTmediated cellular reprogramming [Singh et al., 2016, unpublished]. We have also recently demonstrated that non-integrating adenoviral vectors could be used to robustly induce cellular reprogramming, which is of interest not only because of the clinical relevance of such vectors but also because it reflects the apparently transient requisite period of exposure of target cells to reprogramming agents [Mathison et al., 2016, unpublished].

The first report of utilization of small molecules to induce reprogramming, which were combined with administration of the Yamanaka factor Oct4, targeted epigenetic modulation to enhance reprogramming gene activation and transdifferentiation efficiency [34]. Subsequent studies have utilized chemical cocktails without any transcription factors to induce reprogramming in both murine and human fibroblasts through epigenetic modulation [35, 42]. Such chemically induced cardiomyocyte-like cells (CiCMs) may be a harbinger of future inventive approaches in cardiac reprogramming.

\section{In vivo efficacy of direct cardiac reprogramming}

Perhaps the most convincing evidence of the potential clinical efficacy and relevance of cardiac regeneration through direct cardiac reprogramming lies in in vivo studies, which have reproducibly demonstrated improved ventricular function and reduced fibrosis in rodent infarct models $[7 \cdot \bullet, 8 \cdot, 9-14]$. Although the efficiency of GMT and other reprogramming cocktails in inducing iCM generation in vitro has been reported to be relatively low, intramyocardial injection of these reprogramming factors in infarct rodent models has resulted in approximately a 15-25\% improvement in ejection fraction and $50 \%$ reduction in fibrosis relative to controls $[8 \bullet, 9,10,12-15]$.

Characterization of the regenerated myocardium in fibroblast lineage-tracing models confirmed the fibroblast origin of iCMs, and was found in up to $35 \%$ of fibroblasts in the infarct border zone [8•]. Further analyses has shown that these outcomes were not simply due to the division of pre-existing cardiomyocytes, the fusion of fibroblasts with cardiomyocytes, or the reprogramming of cardiac progenitor cells, which only comprise less than $1 \%$ of the total cells in the heart $[8 \cdot, 10,43]$. These data encouragingly suggest that the in vivo microenvironment is more permissive to cardiac reprogramming than in vitro conditions $[8 \cdot, 9,10,12-15]$. Scar fibroblasts may also be more permissive to transdifferentiation compared to cultured fibroblasts but further investigation is still necessary. Interestingly, a significant but still unexplained reduction in fibrosis which seems to far exceed the extent of iCMgeneration has also been observed in nearly all reprogramming studies reported to date. This observation will be inevitably addressed in future reprogramming studies.

Furthermore, we have shown that prevascularization of the infarcted region (with adenovirus encoding vascular endothelial growth factor [VEGF]) potentiates the effect of reprogramming with GMT, resulting in a larger increase in $\mathrm{EF} \%$ (17 vs $4 \%$ relative to baseline) compared to GMT alone [13]. We have reasoned that such prevascularization brings critical nutrient supply to support the increased metabolic demand of iCMs compared to native fibroblasts in the infarct milieu and thereby supports the survival and function of 
these cells. Subsequent studies have shown that VEGF as well as other angiogenic peptides such as fibroblast growth factor (FGF) activate multiple cardiac transcriptional regulators through modulation of the p38 mitogen-activated protein kinase and phosphoinositol 3kinase/AKT pathways, which may also enhance in vitro reprogramming [37].

\section{Barriers to clinical implementation and future direction}

Although cardiac cellular reprogramming has progressed significantly in recent years, much investigation is still needed prior to clinical implementation. In particular, the recently discovered resistance of human cells to reprogramming is an important barrier to the clinical application of this heart failure treatment strategy [23, 38, 40-42]. The resistance of adult human cardiac fibroblasts to reprogramming compared to rodent cells may be related to the relatively more complex gene regulatory network of the former, which may include rigorous epigenetic barriers to reprogramming that likely normally serve, for example, to impede promiscuous gene activation and tumorigenesis [44]. Systemic gene surveys have for example revealed that over 956 genes encompassing multiple functions such as chromatin regulation, transcription regulation, intracellular signaling, and cell adhesion are opposed to reprogramming in human cells [45].

While overcoming such barriers may be a daunting task, several studies have demonstrated early successes in overcoming these obstacles. In many cases, this barrier has been breached simply by adding additional reprogramming factors to treatment cocktails [23, 38, 40, 41]. More recently, we demonstrated that downregulation of the anti-plasticity factor $p 63$ could be used to enhance reprogramming in murine and human cells, presumably on the basis of the ability to release chromatin-repressed gene activation [Patel et al., 2016, unpublished]. Zhou et al. likewise demonstrated that downregulation of Bmi1 leads to de-repression of key cardio-differentiating genes such as Gata4, Nkx2.5, Isl1, Pitx2, Tbx 20, and Hand2 [36]. Extension of similar strategies to bypass epigenetic barriers to human cellular reprogramming may be expected in future studies.

Without question, innumerable obstacles will need to be overcome before clinical trials of this new strategy might be contemplated. Long-term results regarding iCM survival and sustainable improvements in cardiac function will also need to be investigated. In this context, cardiac reprogramming studies, which have thus far been primarily focused on rodent models, will need to be advanced to a pre-clinical model reflective of the resistance of human cells to reprogramming and of human pathophysiology. Our recent observation that a porcine model might well serve as a surrogate for human studies is encouraging in this regard [Singh et al., 2016, unpublished]. Another potential concern is the risk of arrhythmogenicity due to incomplete or partial transdifferentiation of fibroblasts into iCMs. Although there have been no reports of arrhythmias in the limited number of in vivo studies performed, presumably due to the increased maturation of iCMs in vivo, efforts to enhance reprogramming efficiency and selectively produce ventricular cardiomyocytes over nodal cells or atrial cardiomyocytes are expected to address these concerns [46]. 


\section{Conclusions}

Taken together, evidence from a growing number of studies in vitro and in small animal models suggests that direct cardiac cellular reprogramming offers a highly feasible therapeutic option for the treatment of patients with advanced heart failure. As opposed to the largely unsuccessful strategies of exogenous stem cell implantation or induction of cardiomyocyte proliferation, direct cardiac reprogramming focuses on the abundant population of largely quiescent cardiac fibroblasts found in infarction and border zones to create functional cardiomyocyte-like cells as a means of enhancing post-infarct ventricular function. Given the multifactorial effectors of this strategy, such an approach may well evolve into a multimodal approach involving (1) upregulation of cardio-differentiating factors, (2) downregulation of pro-fibrotic genes, and (3) adjuvant therapy with angiogenic factors to optimize cardiac regeneration. Modulation of epigenetic barriers in human cells will undoubtedly dictate the success of these strategies, but initial results are highly encouraging for a field that is currently barely 6 years old.

\section{Acknowledgments}

This study was funded by the National Heart Lung and Blood Institute (1R01HL121294-01A1 [TR]).

\section{References and Recommended Reading}

Papers of particular interest, published recently, have been highlighted as:

- Of importance

•- Of major importance

1. Baran DA, Jaiswal A. Management of the ACC/AHA Stage D patient: mechanical circulatory support. Cardiol Clin. 2014; 32(1):113-124. viii-ix. [PubMed: 24286582]

2. Kittleson MM, Kobashigawa JA. Management of the ACC/AHA Stage D patient: cardiac transplantation. Cardiol Clin. 2013; 32(1):95-112. viii. [PubMed: 24286581]

3. Fisher SA, Zhang H, Doree C, Mathur A, Martin-Rendon E. Stem cell treatment for acute myocardial infarction. Cochrane Database Syst Rev. 2015; 9:CD006536. This Cochrane review summarizes the results of 41 randomized controlled trials involving bone-marrow stem cell implantation for patients with ischemic heart disease. The difference in left ventricular ejection fraction between the treated and untreated groups was $2-5 \%$, which was determined to not be clinically relevant.

4. Nagalingam RS, Safi HA, Czubryt MP. Gaining myocytes or losing fibroblasts: challenges in cardiac fibroblast reprogramming for infarct repair. J Mol Cell Cardiol. 2015:108-114. [PubMed: 26640115]

5. Sadahiro T, Yamanaka S, Ieda M. Direct cardiac reprogramming: progress and challenges in basic biology and clinical applications. Circ Res. 2015; 116(8):1378-1391. [PubMed: 25858064]

6. Takahashi K, Yamanaka S. Induction of pluripotent stem cells from mouse embryonic and adult fibroblast cultures by defined factors. Cell. 2006; 4:663-676. This historic study established that terminally differentiated cells may be reprogrammed into induced pluripotent stem (iPS) cells using transcription factors Oct4, Sox2, Klf4, and cmyc, ultimately establishing that cellular reprogramming is needed possible. This study preceded the investigation of lineage-based reprogramming for cardiac, hepatic, and neuronal cells.

7. Ieda M, Fu JD, Delgado-Olguin P, Vedantham V, Hayashi Y, Bruneau BG, et al. Direct reprogramming of fibroblasts into functional cardiomyocytes by defined factors. Cell. 2010; 3:375386. Following Yamanaka's methodology, Srivastava and Ieda et al. discovered that defined factors 
Gata4, Mef2c, and Tbx5, which are important in cardiac embryology, may reprogram cardiac fibroblasts into induced cardiomyocytes (iCMs).

8. Qian L, Huang Y, Spencer CI, Foley A, Vedantham V, Liu L, et al. In vivo reprogramming of murine cardiac fibroblasts into induced cardiomyocytes. Nature. 2012; 485(7400):593-598. [PubMed: 22522929] This in vivo extension of Srivastava and Ieda et al. established that GMT administration in infarct murine models results in improved cardiac function and reduced fibrosis.

9. Srivastava D, Ieda M, Fu J, Qian L. Cardiac repair with thymosin $\beta 4$ and cardiac reprogramming factors. Ann N Y Acad Sci. 2012; 1270:66-72. [PubMed: 23050819]

10. Song K, Nam YJ, Luo X, Qi X, Tan W, Huang GN, et al. Heart repair by reprogramming nonmyocytes with cardiac transcription factors. Nature. 2012; 485(7400):599-604. [PubMed: 22660318]

11. Inagawa K, Miyamoto K, Yamakawa H, Muraoka N, Sadahiro T, Umei T, et al. Induction of cardiomyocyte-like cells in infarct hearts by gene transfer of Gata4, Mef2c, and Tbx5. Circ Res. 2012; 111(9):1147-1156. [PubMed: 22931955]

12. Jayawardena TM, Egemnazarov B, Finch EA, Zhang L, Payne JA, Pandya K, et al. MicroRNAmediated in vitro and in vivo direct reprogramming of cardiac fibroblasts to cardiomyocytes. Circ Res. 2012; 110(11):1465-1473. [PubMed: 22539765]

13. Mathison M, Gersch RP, Nasser A, Lilo S, Korman M, Fourman M, et al. In vivo cardiac cellular reprogramming efficacy is enhanced by angiogenic preconditioning of the infarcted myocardium with vascular endothelial growth factor. J Am Heart Assoc. 2012; 1(6):e005652. [PubMed: 23316332]

14. Mathison M, Singh VP, Gersch RP, Ramirez MO, Cooney A, Kaminsky SM, et al. "Triplet" polycistronic vectors encoding Gata4, Mef2c, and Tbx5 enhances postinfarct ventricular functional improvement compared with singlet vectors. J Thorac Cardiovasc Surg. 2014; 148(4):1656.e21664.e2. [PubMed: 24755332]

15. Ma H, Wang L, Yin C, Liu J, Qian L. In vivo cardiac reprogramming using an optimal single polycistronic construct. Cardiovasc Res. 2015; 108(2):217-219. [PubMed: 26400236]

16. Nowbar AN, Mielewczik M, Karavassilis M, Dehbi HM, Shun-Shin MJ, Jones S, et al. Discrepancies in autologous bone marrow stem cell trials and enhancement of ejection fraction (DAMASCENE): weighted regression and meta-analysis. BMJ. 2014; 348:g2688. [PubMed: 24778175]

17. Batty JA, Lima JA, Kunadian V. Direct cellular reprogramming for cardiac repair and regeneration. Eur J Heart Fail. 2015; 18(2):145-156. [PubMed: 26635186] This reference provides an excellent comparison of the various cardiac regeneration strategies.

18. He WJ, Hou Q, Han QW, Han WD, Fu XB. Pluripotent reprogramming and lineage reprogramming: promises and challenges in cardiovascular regeneration. Tissue Eng B Rev. 2013; 20(4):304-313.

19. Ogle BM, Bursac N, Domian I, Huang NF, Menasche P, Murry CE, et al. Distilling complexity to advance cardiac tissue engineering. Sci Transl Med. 2016; 8(342):342ps13.

20. Senyo SE, Steinhauser ML, Pizzimenti CL, Yang VK, Cai L, Wang M, et al. Mammalian heart renewal by preexisting cardiomyocytes. Nature. 2012; 7432:433-436.

21. Sharma A, Zhang Y, Wu SM. Harnessing the induction of cardiomyocyte proliferation for cardiac regenerative medicine. Curr Treat Options Cardiovasc Med. 2015; 17(10):404. [PubMed: 26324824]

22. Kattman SJ, Huber TL, Keller GM. Multipotent flk-1+ cardiovascular progenitor cells give rise to the cardiomyocyte, endothelial, and vascular smooth muscle lineages. Dev Cell. 2006; 11(5):723732. [PubMed: 17084363]

23. Wada R, Muraoka N, Inagawa K, Yamakawa H, Miyamoto K, Sadahiro T, et al. Induction of human cardiomyocyte-like cells from fibroblasts by defined factors. PNAS. 2013; 110(31):1266712672. [PubMed: 23861494]

24. Wang L, Liu Z, Yin C, Asfour H, Chen O, Li Y, et al. Stoichiometry of Gata4, Mef2c, and Tbx5 influences the efficiency and quality of induced cardiac myocyte reprogramming. Circ Res. 2014; 116(2):237-244. [PubMed: 25416133] 
25. Chen JX, Krane M, Deutsch MA, Wang L, Rav-Acha M, Gregoire S, et al. Inefficient reprogramming of fibroblasts into cardiomyocytes using Gata4, Mef2c, and Tbx5. Circ Res. 2012; 111(1):50-55. [PubMed: 22581928]

26. Hirai H, Katoku-Kikyo N, Keirstead SA, Kikyo N. Accelerated direct reprogramming of fibroblasts into cardiomyocyte-like cells with the MyoD transactivation domain. Cardiovasc Res. 2013; 100(1):105-113. [PubMed: 23794713]

27. Zhou H, Dickson ME, Kim MS, Bassel-Duby R, Olson EN. Akt1/protein kinase B enhances transcriptional reprogramming of fibroblasts to functional cardiomyocytes. PNAS. 2015; 112(38): 11864-11869. [PubMed: 26354121]

28. Addis RC, Ifkovits JL, Pinto F, Kellam LD, Esteso P, Rentschler S, et al. Optimization of direct fibroblast reprogramming to cardiomyocytes using calcium activity as a functional measure of success. J Mol Cell Cardiol. 2013; 60:97-106. [PubMed: 23591016]

29. Zhou L, Liu Y, Lu L, Lu X, Dixon RA. Cardiac gene activation analysis in mammalian nonmyoblasic cells by Nkx2-5, Tbx5, Gata4 and Myocd. PLoS One. 2012; 7(10):e48028. [PubMed: 23144723]

30. Protze S, Khattak S, Poulet C, Lindemann D, Tanaka EM, Ravens U. A new approach to transcription factor screening for reprogramming of fibroblasts to cardiomyocyte-like cells. J Mol Cell Cardiol. 2012; 53(3):323-332. [PubMed: 22575762]

31. Christoforou N, Chellappan M, Adler AF, Kirkton RD, Wu T, Addis RC, et al. Transcription factors MYOCD, SRF, Mesp1 and SMARCD3 enhance the cardio-inducing effect of GATA4, TBX5, and MEF2C during direct cellular reprogramming. PLoS One. 2013; 8(5):e63577. [PubMed: 23704920]

32. Ifkovits JL, Addis RC, Epstein JA, Gearhart JD. Inhibition of TGF-beta signaling increases direct conversion of fibroblasts to induced cardiomyocytes. PLoS One. 2014; 9(2):e89678. [PubMed: 24586958]

33. Zhao Y, Londono P, Cao Y, Sharpe EJ, Proenza C, O'Rourke R, et al. High-efficiency reprogramming of fibroblasts into cardiomyocytes requires suppression of pro-fibrotic signalling. Nat Commun. 2015; 10(6):8243.

34. Wang H, Cao N, Spencer CI, Nie B, Ma T, Xu T, et al. Small molecules enable cardiac reprogramming of mouse fibroblasts with a single factor, Oct4. Cell Rep. 2014; 6(5):951-960. [PubMed: 24561253]

35. Fu Y, Huang C, Xu X, Gu H, Ye Y, Jiang C, et al. Direct reprogramming of mouse fibroblasts into cardiomyocytes with chemical cocktails. Cell Res. 2015; 25(9):1013-1024. [PubMed: 26292833]

36. Zhou Y, Wang L, Vaseghi HR, Liu Z, Lu R, Alimohamadi S, et al. Bmi1 is a key epigenetic barrier to direct cardiac reprogramming. Cell Stem Cell. 2016; 3:382-395.

37. Yamakawa H, Muraoka N, Miyamoto K, Sadahiro T, Isomi M, Haginiwa S, et al. Fibroblast growth factors and vascular endothelial growth factor promote cardiac reprogramming under defined conditions. Stem Cell Rep. 2015; 5(6):1128-1142.

38. Muraoka N, Yamakawa H, Miyamoto K, Sadahiro T, Umei T, Isomi M, et al. MiR-133 promotes cardiac reprogramming by directly repressing Snai1 and silencing fibroblast signatures. EMBO J. 2014; 33(14):1565-1581. [PubMed: 24920580]

39. Islas JF, Liu Y, Weng KC, Robertson MJ, Zhang S, Prejusa A, et al. Transcription factors ETS2 and MESP1 transdifferentiate human dermal fibroblasts into cardiac progenitors. PNAS. 2012; 109(32):13016-13021. [PubMed: 22826236]

40. Fu JD, Stone NR, Liu L, Spencer CI, Qian L, Hayashi Y, et al. Direct reprogramming of human fibroblasts toward a cardiomyocyte-like state. Stem Cell Rep. 2013; 1(3):235-247.

41. Nam YJ, Song K, Luo X, Daniel E, Lambeth K, West K, et al. Reprogramming of human fibroblasts toward a cardiac fate. PNAS. 2013; 110(14):5588-5593. [PubMed: 23487791]

42. Cao N, Huang Y, Zheng J, Spencer CI, Zhang Y, Fu JD, et al. Conversion of human fibroblasts into functional cardiomyocytes by small molecules. Science. 2016; 352(6290):1216-1220. [PubMed: 27127239]

43. Wu SM, Chien KR, Mummery C. Origins and fates of cardiovascular progenitor cells. Cell. 2008; 4:537-543. 
44. Ebrahimi B. Reprogramming barriers and enhancers: strategies to enhance the efficiency and kinetics of induced pluripotency. Cell Regeneration (London, England). 2015; 4:10.

45. Qin H, Diaz A, Blouin L, Lebbink RJ, Patena W, Tanbun P, et al. Systematic identification of barriers to human iPSC generation. Cell. 2014; 158(2):449-461. [PubMed: 25036638]

46. Nam YJ, Lubczyk C, Bhakta M, Zang T, Fernandez-Perez A, McAnally J, et al. Induction of diverse cardiac cell types by reprogramming fibroblasts with cardiac transcription factors. Development. 2014; 141(22):4267-4278. [PubMed: 25344074] 


\section{Opinion statement}

Direct cardiac cellular reprogramming of endogenous cardiac fibroblasts directly into induced cardiomyocytes is a highly feasible, promising therapeutic option for patients with advanced heart failure. The most successful cardiac reprogramming strategy will likely be a multimodal approach involving an optimal combination of cardiodifferentiating factors, suppression of fibroblast gene expression, and induction of angiogenic factors. 


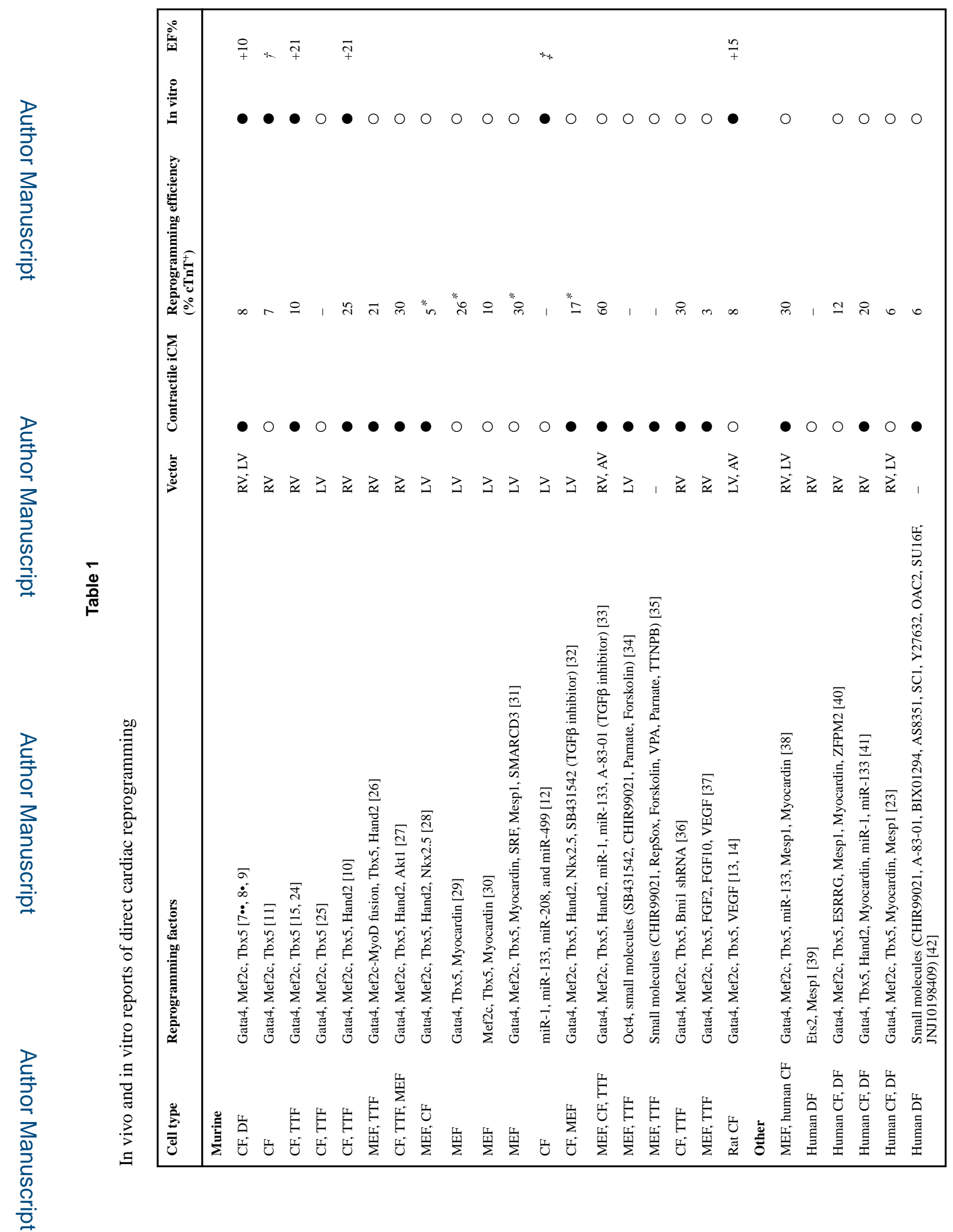

Curr Treat Options Cardiovasc Med. Author manuscript; available in PMC 2017 January 30. 
Patel et al.

Page 12

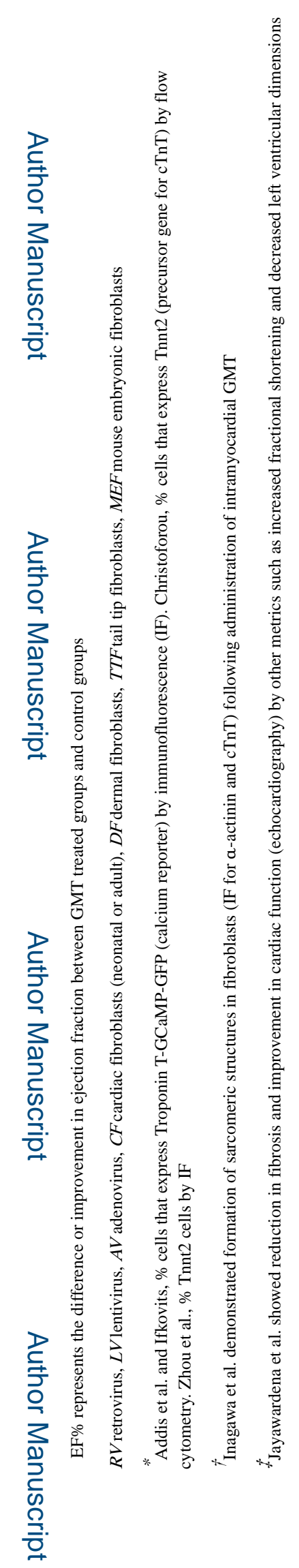

Curr Treat Options Cardiovasc Med. Author manuscript; available in PMC 2017 January 30. 